\title{
Radioiodine Therapy for Thyroid Cancer in the Era of Risk Stratification and Alternative Targeted Therapies
}

\author{
Daniel A. Pryma ${ }^{1}$ and Susan J. Mandel ${ }^{2}$ \\ ${ }^{1}$ Division of Nuclear Medicine and Clinical Molecular Imaging, Department of Radiology, Perelman School of Medicine, University \\ of Pennsylvania, Philadelphia, Pennsylvania; and ${ }^{2}$ Division of Endocrinology, Diabetes and Metabolism, Department of Medicine, \\ Perelman School of Medicine, University of Pennsylvania, Philadelphia, Pennsylvania
}

\begin{abstract}
Learning Objectives: On successful completion of this activity, participants should be able to describe (1) different intents of radioiodine therapy for thyroid cancer; (2) the factors that go into determining that a patient has disease refractory to radioiodine therapy; and (3) approved agents for iodine-refractory thyroid cancer along with emerging targets and treatment strategies.
\end{abstract}

Financial Disclosure: Dr. Pryma is a meeting participant/lecturer for IBA Molecular. The authors of this article have indicated no other relevant relationships that could be perceived as a real or apparent conflict of interest.

CME Credit: SNMMI is accredited by the Accreditation Council for Continuing Medical Education (ACCME) to sponsor continuing education for physicians. SNMMI designates each JNM continuing education article for a maximum of 2.0 AMA PRA Category 1 Credits. Physicians should claim only credit commensurate with the extent of their participation in the activity. For CE credit, SAM, and other credit types, participants can access this activity through the SNMMI website (http://www.snmmilearningcenter.org) through September 2017.

Differentiated thyroid cancers are typically iodine-avid and can be effectively treated with radioiodine. In most patients, radioiodine treatment is done for ablation of residual tissue, and in these cases the focus should be on using the minimum effective dose. Adjuvant therapy can be done to reduce the risk of recurrence, but optimal patient selection and dose are unclear. Patients with advanced disease benefit most from treatment with the maximum-tolerated dose. Recent research has focused on better patient selection and reduced radioiodine doses for remnant ablation. There are emerging targeted therapeutic approaches in patients who are appropriately shown to have iodine-refractory disease, with 1 drug approved by the Food and Drug Administration. Numerous trials are ongoing to assess targeted therapeutics alone or in combination with radioiodine.

Key Words: thyroid cancer; radioiodine therapy; iodine-refractory disease; multitargeted kinases

J Nucl Med 2014; 55:1485-1491

DOI: 10.2967/jnumed.113.131508

$\mathbf{T}$ he use of radioactive iodine for the imaging and therapy of thyroid cancer in many ways represents the birth of nuclear medicine as a specialty. Initial experiences were published in the 1940s, and ${ }^{131}$ I became the first approved radiopharmaceutical by the Food and Drug Administration in 1951. For more than $50 \mathrm{y},{ }^{131} \mathrm{I}$ therapy has been the standard treatment for differentiated thyroid cancer. Over the years, numerous interventions have been considered as potential adjuncts to or replacements for radioactive iodine therapy. The most recent and promising of these include drugs targeted at specific pathways that are aberrant in a substantial percentage of thyroid cancer

For correspondence or reprints contact: Daniel A. Pryma, 3400 Spruce St., Donner 110-C Philadelphia, PA 19104.

E-mail: daniel.pryma@uphs.upenn.edu

Published online Aug. 11, 2014.

COPYRIGHT (C 2014 by the Society of Nuclear Medicine and Molecular Imaging, Inc. patients. The current role of radioactive iodine therapy, including patient selection and preparation and assessment of outcomes, will be reviewed along with the current status of alternate or adjunctive therapeutics.

\section{THYROID CANCER PATHOPHYSIOLOGY, RADIOBIOLOGY, AND DEMOGRAPHICS}

The thyroid gland primarily comprises follicular cells and C cells along with the necessary supporting stroma. The follicular cells take up iodine, synthesizing it into thyroid hormone and subsequently releasing the hormone in response to thyroid-stimulating hormone. These are the cells that can develop into differentiated thyroid cancer and are the chief focus of this article. The $\mathrm{C}$ cells secrete calcitonin and so take part in calcium homeostasis. Medullary thyroid cancer can arise from $\mathrm{C}$ cells; because these cells take no part in the synthesis of iodine-containing thyroid hormone, radioactive iodine therapy plays no role in the treatment of medullary thyroid cancer. Anaplastic cancers can also arise in the thyroid gland. As suggested by the name, the anaplastic cells are essentially completely undifferentiated and do not bear the characteristics of follicular or $\mathrm{C}$ cells. Finally, some conditions result in lymphocytic infiltrates in the thyroid gland (e.g., Hashimoto thyroiditis), and thus lymphoma can arise in the thyroid gland. Most all malignancies arising in the thyroid gland are differentiated thyroid cancers of follicular cell origin.

Differentiated thyroid cancer can be divided into papillary, follicular, and Hürthle cell subtypes. Although the subtypes are assigned by the appearance of the cancer cells and their pattern of growth, it is to be stressed that they all arise from follicular cells. Multiple subtypes and variants can also be described. For example, there are follicular and tall cell variants of papillary thyroid cancer. Some consider Hürthle cell thyroid cancer to be a subtype of follicular thyroid cancer. Some of the variants portend a worse prognosis, whereas others are simply descriptive with no apparent clinical impact.

The hallmark of follicular thyroid cells is the active uptake of iodine from the bloodstream with organification (meaning the iodine has a reasonably long residence time). By substituting radioactive iodide for stable iodide, the behavior of these cells can be exploited 
to both image and deliver therapeutic radiation to follicular thyroid cells. Indeed, most differentiated thyroid cancer cells retain the ability to concentrate and retain iodine.

The fact that radioactive iodine treatment of thyroid cancer is probably the most successful radiopharmaceutical therapy may lead one to suspect that thyroid follicular cells are sensitive to the effects of radiation. However, the definition of radiosensitivity depends on what is being measured. It has been observed that the thyroid gland is among the tissues most likely to develop radiation-induced cancer; thus, it is considered radiosensitive (1). However, when the ability of radiation to kill thyroid cells (a very different definition of radiosensitivity) is being considered, the estimated dose required is hundreds of grays, a radioresistant value (2-5). Thus, in terms of radiotherapy with the intent of killing the target cells, both benign and malignant thyroid cells are quite recalcitrant. The success of radioactive iodine, then, is not a reflection of sensitivity to radiation of the thyroid cells but rather of the ability to deliver hundreds of grays with a reasonably high therapeutic ratio and acceptable normal-tissue toxicity.

\section{CURRENT UTILIZATION OF RADIOACTIVE IODINE THERAPY}

The use of radioactive iodine in thyroid cancer generally falls into 1 of 3 categories: remnant ablation, adjuvant therapy, and cancer treatment. For consistency with guidelines of the American Thyroid Association (ATA), the term used for cancer treatment is radioiodine therapy $(6,7)$. Unless otherwise noted, the principles of treatment discussed in this article follow the 2014 ATA guidelines that were recently publically presented and will be published imminently (7).

Virtually all patients will have some remnant thyroid tissue after thyroidectomy, and it may be desirable to ablate the remnants to simplify future monitoring. If, for example, a patient receives remnant ablation and the thyroglobulin is subsequently undetectable, it is straightforward to monitor the thyroglobulin for increases in the future. If the same patient does not receive remnant ablation and the thyroglobulin rises in the future, it is more difficult to know whether the rise is due to regrowth of normal thyroid tissue or recurrent thyroid cancer. Indeed, recent studies have shown that patients with very low or undetectable serum thyroglobulin in a sensitive assay (functional sensitivity, $0.2 \mathrm{ng} / \mathrm{mL}$ ) at $1 \mathrm{y}$ after treatment have a very low $(\sim 1 \%)$ risk of recurrence in the long term (with mean follow-up currently limited to about 10 y) $(8,9)$. Most patients treated with radioactive iodine today fall into the remnant ablation category. The risk-benefit ratio in such cases is difficult to define, and in some patients remnant ablation is not indicated as discussed further below.

The second category is adjuvant therapy, which mirrors adjuvant treatment in other solid cancers. That is, patients selected for adjuvant therapy have no clinical evidence of residual cancer after resection but are at increased risk for recurrence in the future (i.e., they are suspected to have microscopic residual cancer after surgery). This scenario is considered in patients who have undergone comprehensive resection of extensive locoregional disease without known gross residual cancer. Successful adjuvant treatment will prevent or delay the development of clinically detectable disease (10), though given the long natural history of differentiated thyroid cancer, the available data are incomplete. Of course, patients receiving postoperative adjuvant therapy will also have any remnant ablated with the treatment, but the primary goal of the therapy is adjuvant.

Radioactive iodine therapy is the administration of radioiodine in an attempt to destroy known or suspected active macroscopic viable malignant disease within the patient. This type of use occurs in only a small number of patients referred for radioactive iodine, who represent almost the entire small fraction of thyroid cancer patients who will die of their disease. The framework of therapy in this population must, then, have an approach different from the ablative and adjuvant settings. In the ablative and adjuvant settings, the primary goal is to facilitate monitoring or to decrease the likelihood of a future recurrence (which may still be amenable to curative resection with relatively little morbidity), respectively. Therefore, although there is clearly a benefit to ablative or adjuvant therapy, its magnitude is modest and the focus should be on minimizing toxicity as much as possible to balance it with the magnitude of benefit. In the therapeutic setting, however, the goal is to control a disease that, if not controlled, has a high likelihood of causing death. Thus, the focus should be more on efficacy than toxicity (providing, of course, that toxicity is acceptable). Said more simply, the patient's and treating physician's tolerance for toxicity ought to be greater in the therapeutic setting than in the ablative or adjuvant settings.

\section{PATIENT SELECTION}

Most patients in whom radioiodine therapy is considered will be in the ablative setting, most typically after primary surgery. Considering that most patients have had all discernable macroscopic tumor removed with negative surgical margins, the modest gains from ablation must be balanced against ${ }^{131} \mathrm{I}$ toxicity. The least toxic treatment is no treatment, so the first selection will be determining which patients do not require therapy at all. Guidelines of both the ATA and the National Comprehensive Cancer Network define the population of patients who do not require radioiodine therapy, chiefly those with small $(<1 \mathrm{~cm}$ for papillary and $<2 \mathrm{~cm}$ for follicular or Hürthle cell thyroid cancer) intrathyroidal tumors without evidence of nodal involvement or vascular invasion and with a low postsurgical thyroglobulin level $(6,11)$. Indeed, even patients with multifocal papillary thyroid cancer, in the absence of other risk factors likely do not benefit from remnant ablation (7). There are those patients in whom radioiodine is definitely recommended such as those with large $(>4 \mathrm{~cm})$ primary tumors or gross extrathyroidal extension $(6,11)$.

In between is a large group of patients in whom the appropriateness of radioactive iodine therapy is currently unclear and in whom its use is recommended in selected cases. For example, patients with elevated thyroglobulin without imaging findings of macroscopic disease (on diagnostic whole-body scans, structural imaging, or ${ }^{18} \mathrm{~F}$-FDG PET/CT) often receive empiric radioiodine therapy. There is relatively poor evidence to guide decision making in this area. Guidelines recommend that for patients with relatively low $(<5 \mathrm{ng} / \mathrm{mL})$ and stable serum thyroglobulin, conservative follow-up only is generally recommended, but in the setting of higher serum thyroglobulin or rapidly rising thyroglobulin, empiric therapy may be indicated (7). Although some patients benefit from this empiric therapy, many do not, and it is difficult to balance the risks against the benefits when the likelihood and magnitude of benefit is so poorly understood. Another area of controversy relates to the importance of lateral neck nodal involvement, considered stage I disease in patients younger than $45 \mathrm{y}$ and stage II in patients at least $45 \mathrm{y}$ old. Although there is evidence of the benefit of adjuvant therapy for stage II patients, no benefit has been seen in stage I patients (12). Thus, it may be that some younger patients may not benefit from adjuvant therapy despite locally 
advanced disease, though strong evidence would be needed to withhold treatment in such patients.

Finally, even among patients with high-risk clinical and pathologic features, some have undetectable thyroglobulin after thyroidectomy. Given that low or undetectable thyroglobulin portends an excellent prognosis after remnant ablation $(8,9)$, one could hypothesize that low or undetectable thyroglobulin before radioiodine would predict a low risk of clinically significant disease in the future and that patients with low or undetectable thyroglobulin may not benefit from ablative or adjuvant therapy even with adverse clinical pathologic features. However, currently the utility and role of pretreatment thyroglobulin on decisions of whether to proceed with treatment and dose selection remain unknown.

In some cases, the thyroglobulin level or scan findings can help to determine which patients should or should not receive ablative radioiodine therapy. In many cases, we are left at equipoise with respect to strong (or even moderate or weak) clinical evidence, and thus the decision may be made on the basis of various factors including clinician experience or patient preference. There has been a great deal of interest in the potential of other biomarkers to help predict disease aggressiveness or likelihood of poor outcomes to help make these determinations. Pathophysiologic features such as BRAF V600E, RET oncogene, or RAS mutations have been investigated as potential prognostic biomarkers. Despite early optimism, recent work has not shown strong prognostic ability of these markers $(13,14)$, and their use in deciding treatment is not recommended (7). However, these features may still have implications on therapeutic selection in the setting of advanced disease. As discussed below, multitargeted kinase inhibitors are being tested in advanced thyroid cancer, and a molecular profile of an individual's disease may help to decide the optimal therapeutic regimen. It is to be hoped that ongoing research will continue to help predict the patients in whom radioiodine has a positive risk-benefit ratio.

For radioiodine therapy, initial patient selection is fairly simple and would include essentially any patient with evidence of active distant metastatic disease from differentiated thyroid cancer evident on diagnostic radioiodine scanning. There is, however, a gray area in patients with small-volume disease that may be difficult to detect on imaging who have a negative diagnostic scan result and an even grayer area in patients with biochemical evidence of progressive disease (rising serum thyroglobulin) but negative imaging. Furthermore, in patients with advanced disease who have been previously treated with radioactive iodine, there may come a point when further radioiodine therapy is not warranted; this will be more fully considered later.

\section{DOSE SELECTION}

There are many possible approaches to radioiodine dose selection that are influenced not only by patient factors but also by regulatory restrictions. Again, the principal consideration in the ablative setting is minimizing toxicity while maintaining a reasonable rate of success (though reasonable and success are both quite subjective, leading to the possibility of controversy). The bulk of the evidence in recent years has focused on reducing the dose in lowrisk patients in whom remnant ablation is indicated, where there is fairly strong support that, for example, $1.1 \mathrm{GBq}$ is as effective as $3.7 \mathrm{GBq}$, with a lower level of toxicity (15). Indeed, 2 separate randomized studies published in 2012 showed essentially equivalent ablation success rates whether patients were treated with 1.1 or 3.7 GBq (and whether thyroid hormone withdrawal or recombinant human thyroid-stimulating hormone was used for stimulation); the low-dose group experienced fewer adverse events $(16,17)$. These studies are based largely on the definition of ablation success as normal neck ultrasound findings and low $(<1 \mathrm{ng} / \mathrm{mL})$ thyroglobulin after thyroid-stimulating hormone administration with or without negative results on radioiodine scanning less than $1 \mathrm{y}$ from treatment. The fear, of course, is that the follow-up is insufficient and that in the future the low-dose group may have a higher rate of false-positive thyroglobulin (i.e., thyroglobulin originating from residual normal thyroid tissue). However, it is just as reasonable to fear that with longer follow-up the higher-dose group may have more severe late toxicity. Only time will tell. Unfortunately, currently we will be forced to rely on retrospective evaluations of available data from past patients. Ideally, a randomized trial would be initiated with longer follow-up in the ablative setting and powered to evaluate for recurrence rate in the adjuvant setting; this would require a large number of patients and long follow-up but would provide useful data to the field.

Among adjuvant therapy, the range or potentially recommended doses is wide and the data less strong to help guide choice (7). Many clinicians will choose increasing dose with increasing perceived risk based on clinical and pathologic features (e.g., presence of gross extrathyroidal extension or lateral cervical nodal involvement). In most cases, ablative treatments are limited to the range of 1.1-5.6 GBq. The desire to use the minimum effective dose for adjuvant therapy is similar to that for remnant ablation. However, adjuvant therapy poses a biologic conundrum. $\beta$ particles have a range in tissue on the order of millimeters, and thousands of $\beta$ particles must traverse a cell to provide a high likelihood of lethal DNA damage. For this to be statistically likely, there must be a relatively high number of $\beta$-emitter molecules in and around the tumor cell. When there is a macroscopic focus of disease, $\beta$ particles from various areas overlap and lethal doses are realized. This is why relatively low doses have been shown to be effective in remnant ablation. In microscopic foci of disease, on the other hand, the radiation dose may be insufficient to kill tumor cells because a significant fraction will be deposited outside them $(18,19)$. Therefore, the relatively low but effective remnant ablation doses may be less successful in the adjuvant goal of killing residual microscopic disease. This possibility would imply no benefit for adjuvant radiotherapy, suggesting that, in the absence of residual macroscopic disease with clear iodine uptake, lower radioiodine doses designed for thyroid remnant are sufficient and higher doses should not be used. On the other hand, there are studies indicating reduced local recurrence with postoperative radioiodine ablation $(12,20)$, suggesting a benefit for radioiodine beyond remnant ablation-that is, an adjuvant effect. Resolving this conflict may require a trial in patients with disease at higher risk for local recurrence (nodal metastases, extrathyroidal extension), randomizing patients to low-dose radioiodine for remnant ablation only versus higher doses that may be needed to treat comparatively less iodine-avid and, potentially, smaller residual cancer deposits.

There is a great deal of overlap in dose selection between the adjuvant and radioiodine therapy groups, with therapeutic intent doses frequently in the range of 3.7-7.4 GBq. It is to be stressed that these doses are in a range that is generally considered safe in a typical patient but is not informed by radioiodine kinetics in the particular patient. Factors related to tumor burden, cardiac and renal function, and age, to name a few, can dramatically affect the radioiodine clearance kinetics in a given patient and, therefore, 
can dramatically affect the maximal safe dose. Indeed, among patients with clinically normal renal function, a dose of $7.4 \mathrm{GBq}$ exceeds a dosimetrically derived safe dose in $8 \%-15 \%$ of patients younger than $70 \mathrm{y}$ and $22 \%-38 \%$ of those over the age of $70 \mathrm{y}$ (21).

In the radioiodine therapy setting, the goal, generally, is to give the maximum permissible dose to maximize the probability of controlling the tumor. To achieve this goal while avoiding unacceptable toxicity to organs such as the lungs and bone marrow, dosimetric analysis of radioiodine kinetics in the patient around the time of treatment is required. The whole-body and blood dosimetry that is most widely performed was first described by Benua et al. in 1962 and has been largely unchanged since then (22). It is vitally important to understand that dosimetry determines the maximum dose that can be given safely to prevent severe toxicity (primarily hematologic and pulmonary) and does not inform the likelihood of other toxicities or of the dose delivered to the tumor and likelihood of resultant response.

Radiobiologically, it is possible to construct a tumor control probability curve that gives the likelihood of achieving tumor control at a given radiation dose. A related curve, the normal-tissue toxicity probability curve, can be used to try to find the optimal dose that provides, hopefully, a high likelihood of tumor control and a low likelihood of normal tissue toxicity. Unfortunately, such analyses are not typically available in thyroid cancer. Given the available information, the optimal course of action in a patient with advanced disease is to give the maximum dose that is expected to be in the reasonable range on the normal-tissue toxicity curve with the hope that it is high on the tumor control probability curve. However, currently this can be reliably determined only by the response after therapy.

Developing an understanding of lesional dosimetry would provide critical additional information to help predict which patients will benefit from radioiodine and which are likely to be refractory to therapy despite visible uptake on scanning. There is a positronemitting isotope of iodine ( $\left.{ }^{124} \mathrm{I}\right)$ with a 4.2 -d half-life that is well suited to the kinetics of radioiodine. Numerous investigators have used ${ }^{124}$ I PET or PET/CT not only to permit higher-sensitivity radioiodine imaging but also to allow quantification of uptake in individual lesions to calculate lesional dosimetry (23-27). In the future, it may be possible that ${ }^{124}$ I PET/CT will help reduce the uncertainty in determining which patients have radioiodinerefractory disease to permit patients to receive the most efficacious treatment as soon as possible. For example, an ongoing trial is using ${ }^{124}$ I PET/CT dosimetry to help avoid futile radioiodine therapy (28).

Given the high therapeutic ratio of radioiodine therapy in differentiated thyroid cancer, patients with advanced disease deserve an appropriate attempt at effective radioiodine therapy. The best option would seem to be a dosimetrically derived maximumtolerated dose (with the caveat that patients who had known metastatic disease at the time of a nonmaximal dose and entirely negative results on posttherapy scanning are unlikely to benefit and are usually best served with alternate therapy). Indeed, most patients with advanced thyroid cancer can safely receive more than a typical empiric dose of $7.4 \mathrm{GBq}$ (though in a significant minority such a dose exceeds safe limits) $(21,29)$. Patients with no uptake on a posttherapy scan or obvious progression shortly after a maximal dose can clearly be understood to be iodine-refractory. Those with a clear objective response are likely to benefit. The gray area, then, is when there is a period of stable disease after treatment and it is unclear whether it is a reflection of therapeutic efficacy or simply the natural history of the patient's cancer.

Finally, recently the notion of theranostic agents has become widely discussed. Radioiodine is the prototypical theranostic in that we can be guided by scan findings in addition to other clinical data. A scan that shows clear, intense uptake in all known sites of disease (and, frequently, in previously unknown sites) is one that portends a high likelihood of benefit, whereas entirely negative results on posttherapy scanning nearly guarantee lack of benefit from radioiodine. However, the planar scan typically done provides little to no quantitative information on the radiation dose delivered to sites of tumor and so does not inform as to where the dose delivered is along the tumor control probability curve. Indeed, in a recent study, more than half the patients who had negative results on diagnostic scanning but positive results on posttherapy scanning progressed shortly after receiving radioiodine therapy (30).

\section{IODINE-REFRACTORY DISEASE}

Even with optimized maximum-dose ${ }^{131}$ I therapy, some patients do not achieve tumor control with radioiodine therapy, and these patients are generally labeled iodine-refractory. Because radioiodine is so efficacious in differentiated thyroid cancer, defining what it means to be iodine-refractory is both critically important and quite difficult. However, an objective definition is necessary for uniform care and valid clinical trials. For example, in a recent clinical trial that resulted in the approval of sorafenib for iodinerefractory thyroid cancer patients, iodine-refractory was defined by a combination of factors, including a target lesion without uptake on a radioactive iodine scan (diagnostic or posttherapeutic); progression of disease within 16 mo after radioiodine therapy; multiple previous therapies more than 16 mo beforehand, with progression after at least 2 treatments less than 16 mo apart; and a cumulative radioiodine dose of more than $22.2 \mathrm{GBq}$ (31). Nowhere in the definition is the dose given per treatment considered. It is possible, then, to treat a patient with doses expected to be subtherapeutic and to subsequently label the patient as refractory to the treatment. Imagine, for example, a breast cancer patient with estrogen receptor-positive disease being given a small fraction of the recommended aromatase inhibitor dose and, on progression, being labeled refractory to hormonal therapy. This conclusion does not make biologic sense. Furthermore, in patients with advanced metastatic solid tumors, therapy is not generally given with curative intent. Treatments are, instead, aimed at providing a period of tumor control. Depending on the duration of freedom from progression, a treatment may be considered successful in delaying disease progression, warranting further treatment. For example, in the clinical trial mentioned above, the median progression-free survival was $5.8 \mathrm{mo}$ in patients given placebo and $10.8 \mathrm{mo}$ in those given sorafenib. Consider that a patient who had received radioiodine and achieved a 15-mo progression-free survival would have been considered refractory to radioiodine but subsequently responsive to sorafenib, having achieved a 10.8-mo progressionfree survival. Classifying the patient's response in this manner is not meant to imply that the sorafenib is not effective, only that the definition of iodine-refractory may not be appropriate.

Finally, there is a seemingly arbitrary maximum cumulative dose of $22.2 \mathrm{GBq}$ used in the definition of iodine-refractory in the sorafenib trial (31) and in multiple other clinical trials. There is extensive experience in safely giving cumulative doses in 


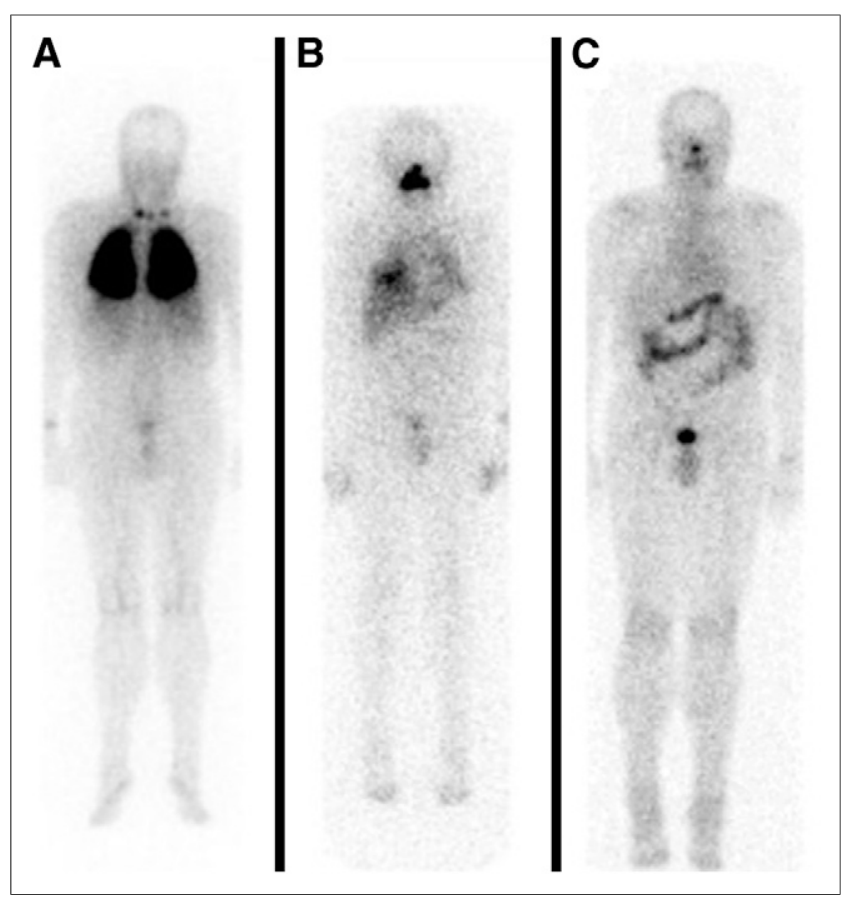

FIGURE 1. A 54-y-old man presented with papillary thyroid cancer metastatic to lungs and lymph nodes. (A) After surgery, dosimetrically derived therapy was done, and anterior posttherapy scan is shown. Multiple subsequent treatments were given for residual disease. (B) Representative anterior posttherapy scan showing residual disease in lungs. (C) Anterior image from final diagnostic scan showing no remaining visible disease. In total, this patient received more than $74 \mathrm{GBq}$ across multiple dosimetric treatments and achieved years of progression-free survival while maintaining acceptable hematologic and lung function.

great excess of this limit without undue toxicity (Fig. 1). Only in cases in which there is a clearly defined cumulative dose toxicity (e.g., anthracyclines) should an effective treatment be withdrawn solely on the basis of cumulative dose. Otherwise, withdrawal should be based on evidence of toxicity or lack of efficacy. The ATA guidelines do not include a cumulative dose in their definition of iodine-refractory (7).

\section{OPTIONS FOR IODINE-REFRACTORY DISEASE}

Once a patient is determined to have iodine-refractory disease, there are multiple potential treatment options. First is the question of whether treatment is required. Even widely metastatic thyroid cancer can be a relatively indolent disease, and in the absence of progressive or symptomatic disease, active surveillance may be preferred over treatment. Furthermore, ${ }^{18}$ F-FDG PET/CT may be helpful to stratify patients, with those who have low ${ }^{18} \mathrm{~F}$-FDG uptake having a favorable prognosis even with iodine-refractory disease (32). In those patients who do require treatment, one approach is to pair a drug aimed at reinducing iodine uptake with radioiodine therapy. Beyond this, numerous pathways have been shown to be aberrant in a significant fraction of patients with iodine-refractory advanced thyroid cancer (Fig. 2), and several therapies targeting these pathways have been tested or are actively being tested in clinical trials.

\section{Reinduction of lodine Uptake}

Given the positive outcomes from radioiodine therapy in patients with iodine-avid disease, an obvious and frequently attempted approach has been to try to increase iodine uptake in disease that is iodine- refractory. Numerous agents have been attempted, including lithium (33-35) and histone deacetylase inhibitors $(36,37)$. Although there were initial reports of efficacy, success appears to have been limited (38).

Recently, however, a study was performed to assess the ability of the mitogen-activated protein kinase kinase (MEK) inhibitor selumetinib to increase iodine uptake in patients with metastatic iodine-refractory disease (39). The investigators found that 12 of 20 evaluable patients had an increase in iodine uptake in their disease. An exciting aspect of the trial was the use of ${ }^{124}$ I PET/CT as an integral biomarker-only patients whose scans showed an expected dose of at least 20 Gy to sites of disease went on to therapy. Thus, 8 of 12 patients went on to treatment, and all had biochemical evidence of response.

The magnitude and reproducibility of this effect will need to be confirmed in larger studies. However, multiagent combination therapy has become the norm in most therapeutic approaches to solid tumors, and it is likely that radioiodine therapy could have a synergistic or additive effect when combined with additional therapeutic agents. There is an ongoing phase III study using selumetinib in combination with adjuvant radioiodine in high-risk patients after thyroidectomy (40); thus, there is a potential for combination therapy in both the adjuvant and the therapeutic settings.

\section{Multitargeted Kinase Inhibitors}

Multiple signaling pathways are active in tumor deposits that are critical in cancer cell proliferation, growth, and invasion as well as in promoting the stroma and vascularity that these growing tumor deposits need to survive. Several of these pathways have been identified (Fig. 2) and targeted therapies developed. The pathways most often exploited in thyroid cancer include BRAF, RET, ERK (extracellular signal-regulated kinase), MET, and other antiangiogenic treatments. Many of these treatments are in a class of drugs called multitargeted kinase inhibitors. One of these, sorafenib, inhibits multiple tyrosine and Raf kinases $(41,42)$ and was approved for use in iodine-refractory metastatic thyroid cancer in late 2013 (31). As discussed above, the basis for approval was an improved median progression-free survival of 10.8 mo in patients treated with sorafenib versus 5.8 mo in patients who received placebo. A search of clinicaltrials.gov reveals many ongoing studies of targeted therapeutic agents in iodinerefractory thyroid cancer, indicating the need in this area (43-46).

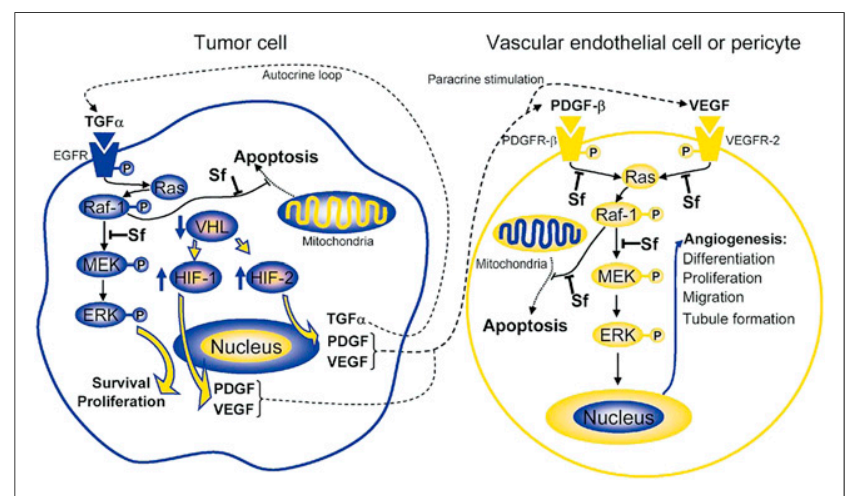

FIGURE 2. Various signaling pathways in cancers that are amenable to targeted therapies. Sorafenib has inhibitory effects on multiple pathways. EGFR = endothelial growth factor receptor; HIF-1 = hypoxiainducible factor-1; HIF-2 = hypoxia-inducible factor-2; PDGF = plateletderived growth factor; SF = sorafenib; TFGa = tumor growth factor $\alpha$; VEGF = vascular endothelial growth factor; $\mathrm{VHL}=$ von Hippel Lindau. (Reprinted with permission of (54).) 
Many of the pathophysiologic biomarkers previously tested and largely rejected as potential prognostic biomarkers may yet have a role as predictive biomarkers. Because of the targeted nature of the therapeutics being tested, it is to be expected that a given patient may be more likely to respond to one drug over another based on pathophysiologic features of his or her disease. Ideally, such therapeutics will be paired with diagnostic tests to help choose the optimal treatment for any patient. For example, selumetinib (discussed above in combination with radioiodine) has been tested as a single-agent treatment in iodine-refractory disease (47). This phase II trial showed a trend toward longer progression-free survival in the patients with a BRAF V600E mutation. Most studies of targeted therapeutics in iodine-refractory thyroid cancer include analysis stratified by putative predictive biomarkers such as BRAF mutation status.

Although there is optimism that targeted therapeutics beyond radioiodine may be effective in (iodine-refractory) thyroid cancer, these agents also have drawbacks. In contrast to radioiodine therapy that is given at intervals of months to years, these targeted agents are given regularly, often a daily or twice daily oral pill. Toxicity is common and can have a significant impact on quality of life; life-threatening toxicity is less common but possible (48). For example, many of these treatments cause rash, hand and foot desquamation, diarrhea, and asthenia, and the toxicities frequently occur early in the treatment regimen rather than as late cumulative events (48). This consideration is particularly important in patients who are often asymptomatic from their cancer. Furthermore, targeted therapies broadly have been shown to eventually lose efficacy due to a variety of escape mechanisms (49-52). That is, whereas the drug continues to block its target, the cancer finds a way to escape the inhibitory effect on its growth. With many of the daily oral targeted therapeutics, this escape occurs within a matter of months in most patients. Taken in context, this could be a helpful result or a relatively small benefit. For example, patients with metastatic renal cell or hepatocellular cancer could have a dramatic increase in duration of survival from a few months of progression-free survival induced by one of these targeted therapies. In the more protracted timeline of advanced thyroid cancer, though, a few months of improvement would be expected to have less of an impact on the percentage improvement on duration of survival. Finally, given that these treatments are largely cytostatic rather than cytotoxic, evaluating response to therapy is difficult (as discussed above, it is difficult to know if stable disease is a reflection of treatment efficacy or the natural history of the cancer). Radioiodine scanning will not be particularly informative because these patients, by definition, have iodine-refractory disease. However, other molecular imaging studies, for example ${ }^{18}$ F-FDG PET/CT (53), may be helpful to differentiate responsive patients from nonresponsive patients with anatomically stable disease.

\section{CONCLUSION}

Radioiodine is a prototypical theranostic agent permitting both imaging and therapy. The therapeutic use of radioiodine continues to be refined with a better understanding of the risks and benefits of therapy. It is hoped that this refinement will permit decreased (or absent) dose (and decreased toxicity) in the patients who are destined to do well, increased dose in the patients who will benefit from treatment, and more appropriate discontinuation or modification of therapy in those unlikely to benefit from single-agent radioiodine therapy. ${ }^{124}$ I PET/CT imaging may help physicians make rational decisions on the likelihood of response to therapy. Tar- geted therapeutics, alone or in combination with radioactive iodine, may play a role in both adjuvant and advanced disease settings. Nuclear medicine physicians should play a key consultative role in the treating team to optimize radioiodine therapy and confirm the presence or absence of iodine-refractory disease.

Multiple targeted therapeutics are being tested in the setting of iodine-refractory thyroid cancer, with one agent now approved in the United States. Although it is excellent for patients with iodine-refractory disease to have options, it is critically important to recognize that radioiodine is an effective treatment in a large subset of patients with metastatic differentiated thyroid cancer and should not be abandoned prematurely (nor should it be used when there is evidence of its futility).

The future of therapy in advanced differentiated thyroid cancer is likely to include multiagent treatment. Radioiodine may play an important role as a component of such therapeutic approaches.

\section{ACKNOWLEDGMENT}

We acknowledge David A. Mankoff for his valuable input.

\section{REFERENCES}

1. Rall JE. The effects of radiation on the thyroid gland: a quantitative analysis. Prog Clin Biol Res. 1981;74:29-43.

2. Maxon HR III, Englaro EE, Thomas SR, et al. Radioiodine-131 therapy for welldifferentiated thyroid cancer: a quantitative radiation dosimetric approach-outcome and validation in 85 patients. J Nucl Med. 1992;33:1132-1136.

3. Maxon HR, Thomas SR, Hertzberg VS, et al. Relation between effective radiation dose and outcome of radioiodine therapy for thyroid cancer. $N$ Engl J Med. 1983;309:937-941.

4. Maxon HR, Thomas SR, Samaratunga RC. Dosimetric considerations in the radioiodine treatment of macrometastases and micrometastases from differentiated thyroid cancer. Thyroid. 1997;7:183-187.

5. Schlumberger MJ. Papillary and follicular thyroid carcinoma. $N$ Engl J Med. 1998;338:297-306.

6. Cooper DS, Doherty GM, Haugen BR, et al. Revised American Thyroid Association management guidelines for patients with thyroid nodules and differentiated thyroid cancer. Thyroid. 2009;19:1167-1214.

7. Haugen BR, Mandel SJ, Nikiforov Y, et al. 2014 American Thyroid Association (ATA) guidelines on thyroid nodules and differentiated thyroid cancer: highlights, consensus, and controversies. Paper presented at: 16th International Congress of Endocrinology; June 20, 2014; Chicago, IL.

8. Durante C, Montesano T, Torlontano M, et al. Papillary thyroid cancer: time course of recurrences during postsurgery surveillance. J Clin Endocrinol Metab. 2013;98:636-642.

9. Han JM, Kim WB, Yim JH, et al. Long-term clinical outcome of differentiated thyroid cancer patients with undetectable stimulated thyroglobulin level one year after initial treatment. Thyroid. 2012;22:784-790.

10. Mazzaferri EL. Thyroid remnant ${ }^{131}$ I ablation for papillary and follicular thyroid carcinoma. Thyroid. 1997;7:265-271.

11. Thyroid carcinoma (version 2.2013). National Comprehensive Cancer Network website. http://www.nccn.org/professionals/physician_gls/pdf/thyroid.pdf. Accessed July 29, 2014.

12. Jonklaas J, Sarlis NJ, Litofsky D, et al. Outcomes of patients with differentiated thyroid carcinoma following initial therapy. Thyroid. 2006;16:1229-1242.

13. Barbaro D, Incensati RM, Materazzi G, et al. The BRAF V600E mutation in papillary thyroid cancer with positive or suspected pre-surgical cytological finding is not associated with advanced stages or worse prognosis. Endocrine. 2014;45:462-468.

14. Stanojevic B, Dzodic R, Saenko V, et al. Mutational and clinico-pathological analysis of papillary thyroid carcinoma in Serbia. Endocr J. 2011;58:381-393.

15. Cheng W, Ma C, Fu H, et al. Low- or high-dose radioiodine remnant ablation for differentiated thyroid carcinoma: a meta-analysis. J Clin Endocrinol Metab. 2013; 98:1353-1360.

16. Schlumberger M, Catargi B, Borget I, et al. Strategies of radioiodine ablation in patients with low-risk thyroid cancer. $N$ Engl J Med. 2012;366: 1663-1673.

17. Mallick U, Harmer C, Yap B, et al. Ablation with low-dose radioiodine and thyrotropin alfa in thyroid cancer. N Engl J Med. 2012;366:1674-1685. 
18. Grosev D, Loncaric S, Huic D, Dodig D. Geometric models in dosimetry of thyroid remnant mass. Nuklearmedizin. 2008;47:120-126.

19. Van Nostrand D. The benefits and risks of I-131 therapy in patients with welldifferentiated thyroid cancer. Thyroid. 2009;19:1381-1391.

20. Chow SM, Yau S, Kwan CK, Poon PC, Law SC. Local and regional control in patients with papillary thyroid carcinoma: specific indications of external radiotherapy and radioactive iodine according to T and N categories in AJCC 6th ed. Endocr Relat Cancer. 2006;13:1159-1172.

21. Tuttle RM, Leboeuf R, Robbins RJ, et al. Empiric radioactive iodine dosing regimens frequently exceed maximum tolerated activity levels in elderly patients with thyroid cancer. J Nucl Med. 2006;47:1587-1591.

22. Benua RS, Cicale NR, Sonenberg M, Rawson RW. The relation of radioiodine dosimetry to results and complications in the treatment of metastatic thyroid cancer. AJR. 1962;87:171-182.

23. Freudenberg LS, Jentzen W, Gorges R, et al. ${ }^{124}$ I-PET dosimetry in advanced differentiated thyroid cancer: therapeutic impact. Nuklearmedizin. 2007;46:121-128.

24. Pettinato C, Monari F, Nanni C, et al. Usefulness of ${ }^{124} \mathrm{I}$ PET/CT imaging to predict absorbed doses in patients affected by metastatic thyroid cancer and treated with ${ }^{131}$ I. Q J Nucl Med Mol Imaging. 2012;56:509-514.

25. Sgouros G, Hobbs RF, Atkins FB, Van Nostrand D, Ladenson PW, Wahl RL. Three-dimensional radiobiological dosimetry (3D-RD) with ${ }^{124}$ I PET for ${ }^{131}$ I therapy of thyroid cancer. Eur J Nucl Med Mol Imaging. 2011;38(suppl 1):S41-S47.

26. Sgouros G, Kolbert KS, Sheikh A, et al. Patient-specific dosimetry for ${ }^{131} \mathrm{I}$ thyroid cancer therapy using ${ }^{124} \mathrm{I}$ PET and 3-dimensional-internal dosimetry (3D-ID) software. J Nucl Med. 2004;45:1366-1372.

27. Westphal JG, Winkens T, Kuhnel C, Freesmeyer M. Low-activity I-PET/lowdose CT versus I probe measurements in pretherapy assessment of radioiodine uptake in benign thyroid diseases. J Clin Endocrinol Metab. 2014;99:2138-2145.

28. Kist JW, de Keizer B, Stokkel MP, Hoekstra OS, Vogel WV; THYROPET study group. Recurrent differentiated thyroid cancer: towards personalized treatment based on evaluation of tumor characteristics with PET (THYROPET Study): study protocol of a multicenter observational cohort study. BMC Cancer. 2014; $14: 405$.

29. Van Nostrand D, Atkins F, Moreau S, et al. Utility of the radioiodine whole-body retention at 48 hours for modifying empiric activity of 131-iodine for the treatment of metastatic well-differentiated thyroid carcinoma. Thyroid. 2009;19: 1093-1098.

30. Sabra MM, Grewal RK, Tala H, Larson SM, Tuttle RM. Clinical outcomes following empiric radioiodine therapy in patients with structurally identifiable metastatic follicular cell-derived thyroid carcinoma with negative diagnostic but positive post-therapy ${ }^{131}$ I whole-body scans. Thyroid. 2012;22:877-883.

31. Nexavar (sorafenib) tablets, oral prescribing information. Daily Med website. http://dailymed.nlm.nih.gov/dailymed/lookup.cfm?setid=b50667e4-5ebc-4968a646-d605058dbef0. Accessed July 29, 2014.

32. Wang W, Larson SM, Fazzari M, et al. Prognostic value of $\left[{ }^{18} \mathrm{~F}\right]$ fluorodeoxyglucose positron emission tomographic scanning in patients with thyroid cancer. $J$ Clin Endocrinol Metab. 2000;85:1107-1113.

33. Koong SS, Reynolds JC, Movius EG, et al. Lithium as a potential adjuvant to ${ }^{131} \mathrm{I}$ therapy of metastatic, well differentiated thyroid carcinoma. J Clin Endocrinol Metab. 1999;84:912-916.

34. Pons F, Carrio I, Estorch M, Ginjaume M, Pons J, Milian R. Lithium as an adjuvant of iodine-131 uptake when treating patients with well-differentiated thyroid carcinoma. Clin Nucl Med. 1987;12:644-647.

35. Yamazaki CA, Padovani RP, Biscolla RP, et al. Lithium as an adjuvant in the postoperative ablation of remnant tissue in low-risk thyroid carcinoma. Thyroid. 2012;22:1002-1006.

36. Furuya F, Shimura H, Suzuki H, et al. Histone deacetylase inhibitors restore radioiodide uptake and retention in poorly differentiated and anaplastic thyroid cancer cells by expression of the sodium/iodide symporter thyroperoxidase and thyroglobulin. Endocrinology. 2004;145:2865-2875.

37. Kitazono M, Robey R, Zhan Z, et al. Low concentrations of the histone deacetylase inhibitor, depsipeptide (FR901228), increase expression of the $\mathrm{Na}(+) / \mathrm{I}(-)$ symporter and iodine accumulation in poorly differentiated thyroid carcinoma cells. J Clin Endocrinol Metab. 2001;86:3430-3435.

38. Liu YY, van der Pluijm G, Karperien M, et al. Lithium as adjuvant to radioiodine therapy in differentiated thyroid carcinoma: clinical and in vitro studies. Clin Endocrinol (Oxf). 2006;64:617-624.

39. Ho AL, Grewal RK, Leboeuf R, et al. Selumetinib-enhanced radioiodine uptake in advanced thyroid cancer. $N$ Engl J Med. 2013;368:623-632.

40. Study comparing complete remission after treatment with selumetinib/placebo in patient with differentiated thyroid cancer (ASTRA). ClinicalTrials.gov website. http://www.clinicaltrials.gov/ct2/show/NCT01843062?term=selumetinib\&rank=17. Accessed July 29, 2014.

41. Smalley KS, Xiao M, Villanueva J, et al. CRAF inhibition induces apoptosis in melanoma cells with non-V600E BRAF mutations. Oncogene. 2009;28:85-94.

42. Wilhelm SM, Adnane L, Newell P, Villanueva A, Llovet JM, Lynch M. Preclinical overview of sorafenib, a multikinase inhibitor that targets both Raf and VEGF and PDGF receptor tyrosine kinase signaling. Mol Cancer Ther. 2008;7:3129-3140.

43. Aflibercept in treating patients with recurrent and/or metastatic thyroid cancer that did not respond to radioactive iodine therapy. ClinicalTrials.gov website. http://www.clinicaltrials.gov/ct2/show/NCT00729157?term $=$ NCT00729157\& rank=1. Accessed July 29, 2014.

44. Combination of temsirolimus and sorafenib in the treatment of radioactive iodine refractory thyroid cancer. ClinicalTrials.gov website. http://www.clinicaltrials.gov/ ct2/show/NCT01025453?term $=$ NCT01025453\&rank=1. Accessed July 29, 2014.

45. Evaluation of efficacy, safety of vandetanib in patients with differentiated thyroid cancer (VERIFY). ClinicalTrials.gov website. http://www.clinicaltrials.gov/ct2/ show/NCT01876784?term $=$ NCT01876784\&rank=1. Accessed July 29, 2014.

46. A multicenter, randomized, double-blind, placebo-controlled, phase 3 trial of lenvatinib (E7080) in ${ }^{131}$ I-refractory differentiated thyroid cancer. ClinicalTrials. gov website. http://www.clinicaltrials.gov/ct2/show/NCT01321554?term =NCT0 1321554\&rank=1. Accessed July 29, 2014.

47. Hayes DN, Lucas AS, Tanvetyanon T, et al. Phase II efficacy and pharmacogenomic study of selumetinib (AZD6244; ARRY-142886) in iodine-131 refractory papillary thyroid carcinoma with or without follicular elements. Clin Cancer Res. 2012;18:2056-2065.

48. Brose MS, Frenette CT, Keefe SM, Stein SM. Management of sorafenib-related adverse events: a clinician's perspective. Semin Oncol. 2014;41(suppl 2):S1-S16.

49. Motzer RJ, Porta C, Vogelzang NJ, et al. Dovitinib versus sorafenib for third-line targeted treatment of patients with metastatic renal cell carcinoma: an openlabel, randomised phase 3 trial. Lancet Oncol. 2014;15:286-296.

50. Tejpar S, Prenen H, Mazzone M. Overcoming resistance to antiangiogenic therapies. Oncologist. 2012;17:1039-1050.

51. Sampson JH, Heimberger AB, Archer GE, et al. Immunologic escape after prolonged progression-free survival with epidermal growth factor receptor variant III peptide vaccination in patients with newly diagnosed glioblastoma. J Clin Oncol. 2010;28:4722-4729.

52. Weinstein IB, Joe A. Oncogene addiction. Cancer Res. 2008;68:3077-3080, discussion 3080 .

53. Carr LL, Mankoff DA, Goulart BH, et al. Phase II study of daily sunitinib in FDG-PET-positive, iodine-refractory differentiated thyroid cancer and metastatic medullary carcinoma of the thyroid with functional imaging correlation. Clin Cancer Res. 2010;16:5260-5268.

54. Gollob JA, Wilhelm S, Carter C, Kelley SL. Role of Raf kinase in cancer: therapeutic potential of targeting the Raf/MEK/ERK signal transduction pathway. Semin Oncol. 2006;33:392-406. 